\title{
U-transformation-finite element method in 3-dimensional analysis of orthotropic laminates
}

\author{
P. Yan ${ }^{a}$, F.L. Chen ${ }^{a}$, C.P. Jiang ${ }^{\mathrm{a}, \mathrm{b}, *}$, F. Song ${ }^{\mathrm{b}}$ \\ ${ }^{a}$ School of Aeronautic Science and Engineering, Beijing University of Aeronautics and Astronautics, 100191 Beijing, China \\ ${ }^{\mathrm{b}}$ State Key Laboratory of Nonlinear Mechanics (LNM), Institute of Mechanics, Chinese Academy of Sciences, 100190 Beijing, China
}

\section{A R T I C L E I N F O}

\section{Article history:}

Available online 24 May 2010

\section{Keywords:}

U-transformation

Finite element method

Laminates

Composites

\begin{abstract}
A B S T R A C T
For an orthotropic laminate, an equivalent system with doubly cyclic periodicity is introduced. Then a 3-dimensional finite element model for the equivalent system is transformed into the unitary space, where the large finite element matrix equation is decoupled into some small matrix equations. Such a decoupling very efficiently reduces the computational effort. For an orthotropic laminate with four clamped edges, no exact elasticity solution is available, and the deflection values predicted by different methods have a considerable difference each other for a small length-to-thickness ratio. The present predictions are the largest because the present method is a full 3-dimensional finite element analysis without superfluous constraints. Illustrative numerical examples are presented to observe the distributions of stresses through the thickness of the laminates.
\end{abstract}

(C) 2010 Elsevier Ltd. All rights reserved.

\section{Introduction}

From aircraft and aerospace industry, automobile industry to building structures, applications of composite laminates have expanded rapidly in the recent decades. The classical laminate theory [1], the first-order [2,3] and higher-order [4,5] shear deformation laminate theories are 2-dimensional (2-D) ones, which are referred to as equivalent single layer (ESL) theories, and have been reported to predict the overall response like gross deformations, buckling modes, inplane stresses and so on reasonably well. The early use of composite laminates was mainly restricted to secondary structural components, and the 2-D ESL theories often prove adequate.

However, as laminated composite materials undergo the transition from secondary structural components to primary critical structural components, the goals of analysis must be broadened to include a highly accurate assessment of localized regions where damage initiation is likely [6]. For example, the key component principle wing box in a Boeing B-787 is first made of composites (Carbon/epoxy) [7], so the more accurate stress analysis is needed. In such cases, the simple 2-D ESL theories are of limited value since they are based on the simple displacement pattern assumption through the thickness, and fail to capture the transverse/interlaminar stresses, which may lead to delamination, one of the major failure modes of composite laminates. So the more accurate 2-D

\footnotetext{
* Corresponding author at: School of Aeronautic Science and Engineering, Beijing University of Aeronautics and Astronautics, 100191 Beijing, China. Tel.: +86 010 82317508.

E-mail address: jiangchiping@buaa.edu.cn (C.P. Jiang).
}

ESL theories [8-10] and layerwise laminate theories [11-13], even the 3-D analysis theories are very necessary. An accurate 3-D analysis not only can be essential prerequisite for understanding the failure behavior, but also can be the benchmark for the 2-D ESL theories and the layerwise laminate theories.

Because of mathematical difficulties involved, 3-D exact elasticity solutions are very limited. Pagano [14] presented such a benchmark problem, i.e. a three-layer equi-thickness cross-ply square laminate with four simply supported edges under a bi-sinusoidal transverse load on the top surface. However, for a thick laminate with other boundary conditions, for example four clamped edges, different methods provide very different results (refer to Example 2 in Section 5). The theories and computational methods for composite laminates have been an active research area in recent decades [6]. For example, Cheung and Jiang [15] presented a finite layer method in 3-D analysis of composite laminates. Attallah et al. [16] introduced a combined finite strip and state space approach to obtain 3-D solutions of laminated composite plates with simply supported ends. Bambole and Desai [17] presented a 27-node 3-D hexahedral hybrid-interface finite element model for thick laminated composite plates. Kant et al. [18] presented a semi-analytical method with mixed variables (displacements and transverse stresses) for composite and sandwich laminates.

The finite element method is a very powerful and versatile tool of solution. However, as the inplane size (length and width) of a lamina is much larger than the thickness, to ensure comparable three dimensions of a cuboidal element, the inplane mesh must be very dense, which yields a very large finite element matrix equation, hence a very expensive and time-consuming computational effort. 
It is observed that for a rectangular laminate, an inplane uniform mesh yields numerous doubly periodic repeated cuboidal elements, which contain numerous identical pieces of information. If they are decoupled, the computational effort will very greatly decrease. Such a decoupling can be accomplished by using the Utransformation $[19,20]$. If a structure consists of cyclic periodic substructure, the finite element total stiffness matrix becomes a quasi-diagonal matrix, which can be decoupled into individual small matrixes in the unitary space. Such a decoupling is the purpose of the present work. A full 3-D finite element method, i.e. the U-transformation-finite element method for orthotropic laminates is developed.

This paper is organized as follows. First a 3-D finite element model and the equivalent cyclic periodic structure of a composite laminate are introduced in Section 2. Then the characteristic of the stiffness matrix for the equivalent structure is described, and the decoupling of the finite element equations using the double U-transformation is dealt with in Section 3. The detailed expressions of the load vector for different boundary conditions are given in Section 4. Finally, numerical examples and a comparison with available results are presented and discussed in Section 5.

\section{Three-dimensional finite element model with a cyclic periodicity}

The U-transformation-finite element method can decouple the computational region of the whole laminate into individual subregions in the unitary space, and the prerequisite is that the elements possess a cyclic periodicity. This section focuses on the construction of a 3-D finite element model with a cyclic periodicity in two directions for an orthotropic laminate.

A rectangular cross-ply laminate consisting of $N$ orthotropic lamina is shown in Fig. 1. The laminate is uniformly discretized into $m \times n \times L$ 3-D elements as shown in Fig. 2a, where each lamina is discretized into $l_{i}(i=1,2, \ldots, N)$ sublayers and $L=\sum_{i=1}^{N} l_{i}$.

Referring to the fiber-matrix coordinate system $(1,2,3)$ in Fig. 1, the compliance matrix $\mathbf{S}$ and the stiffness matrix $\mathbf{C}$ for a lamina can be written as

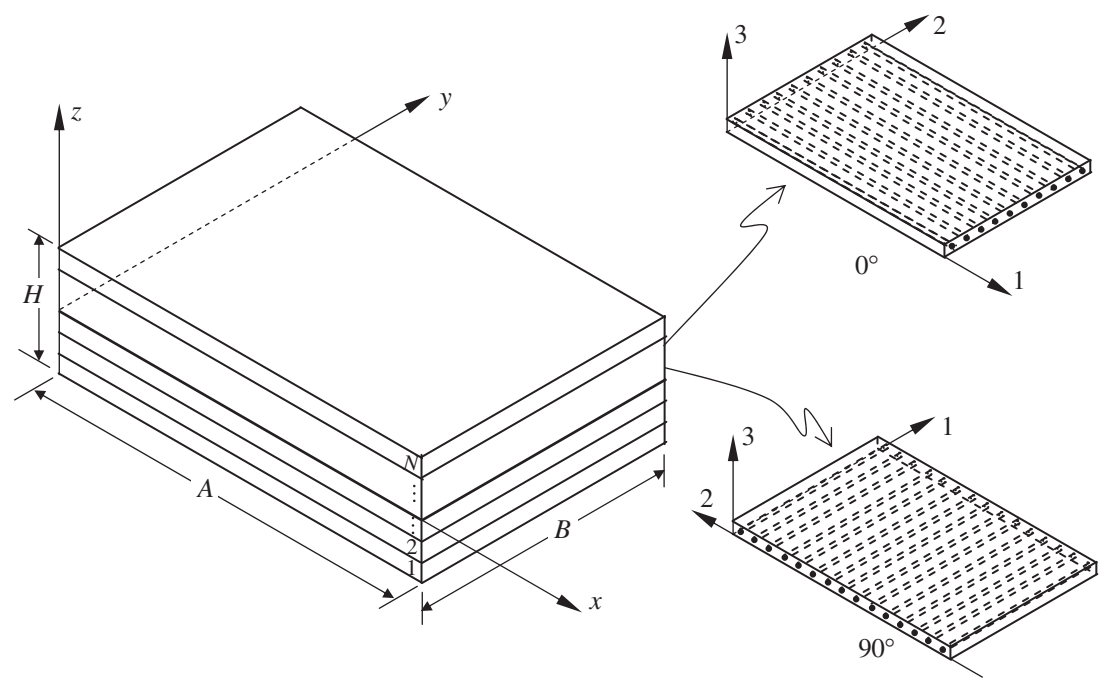

Fig. 1. A $N$-layer rectangular orthotropic laminate and its laminate coordinate system and fiber-matrix coordinate system.

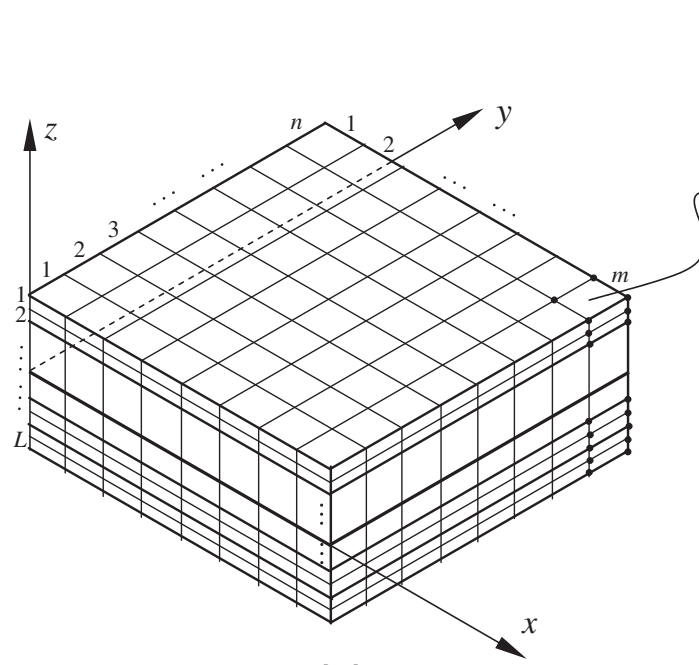

(a)

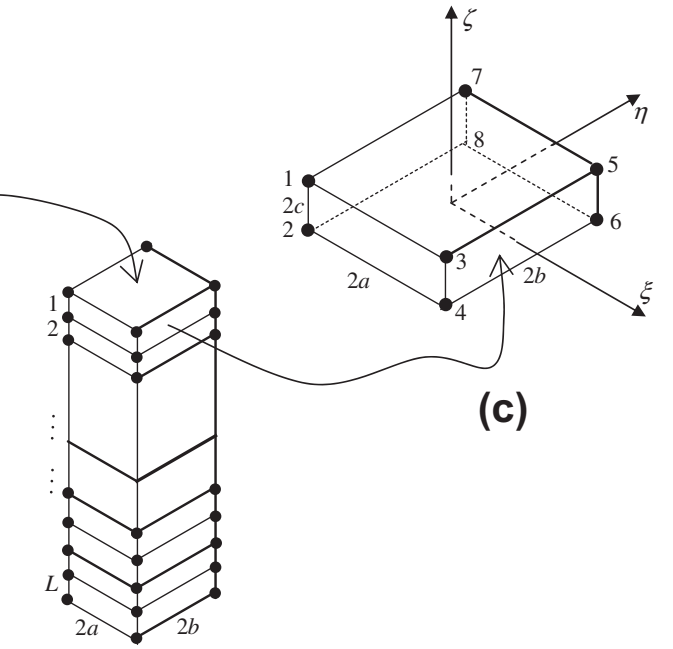

(b)

Fig. 2. Finite element model for a laminate: (a) 3-D cubic mesh, (b) a column of elements, (c) a 8-node element. 
$\mathbf{S}=\left[\begin{array}{cccccc}\frac{1}{E_{1}} & -\frac{v_{21}}{E_{2}} & -\frac{v_{31}}{E_{3}} & 0 & 0 & 0 \\ -\frac{v_{12}}{E_{1}} & \frac{1}{E_{2}} & -\frac{v_{32}}{E_{3}} & 0 & 0 & 0 \\ -\frac{v_{13}}{E_{1}} & -\frac{v_{23}}{E_{2}} & \frac{1}{E_{3}} & 0 & 0 & 0 \\ 0 & 0 & 0 & \frac{1}{G_{23}} & 0 & 0 \\ 0 & 0 & 0 & 0 & \frac{1}{G_{31}} & 0 \\ 0 & 0 & 0 & 0 & 0 & \frac{1}{G_{12}}\end{array}\right]$

$\mathbf{C}=\mathbf{S}^{-1}$

Then the stiffness matrix in the laminate coordinates $(x, y, z)$ in Fig. 1 can be obtained by a rotation of $90^{\circ}$ or $0^{\circ}$.

Adopt a column of 8-node Wilson finite elements [21] as shown in Fig. $2 \mathrm{~b}$ and c. The column of elements can be regarded as an equivalent 2-D 4-node element, where each node is of $3(L+1)$ degrees of freedom as shown in Fig. 3. The displacement vector and load vector of the equivalent node (EN) are denoted by $\{\delta\}_{k}^{\mathrm{EN}}$ and $\{F\}_{k}^{\mathrm{EN}}(k=1,2,3,4)$, respectively. The displacement vector and load vector of the equivalent 4-node element can be written as

$\{\delta\}^{\mathrm{EE}}=\left\{\left(\{\delta\}_{1}^{\mathrm{EN}}\right)^{\mathrm{T}},\left(\{\delta\}_{2}^{\mathrm{EN}}\right)^{\mathrm{T}},\left(\{\delta\}_{3}^{\mathrm{EN}}\right)^{\mathrm{T}},\left(\{\delta\}_{4}^{\mathrm{EN}}\right)^{\mathrm{T}}\right\}$,

$\{F\}^{\mathrm{EE}}=\left\{\left(\{F\}_{1}^{\mathrm{EN}}\right)^{\mathrm{T}},\left(\{F\}_{2}^{\mathrm{EN}}\right)^{\mathrm{T}},\left(\{F\}_{3}^{\mathrm{EN}}\right)^{\mathrm{T}},\left(\{F\}_{4}^{\mathrm{EN}}\right)^{\mathrm{T}}\right\}$

where the superscript "EE" refers to the equivalent 4-node element and the superscript " $T$ " denotes the transpose. Correspondingly, the stiffness matrix of the equivalent 4-node element is denoted by $[K]^{\mathrm{EE}}$, which is a $12(L+1) \times 12(L+1)$ matrix.

Three laminates with different boundary conditions are shown in Fig. 4, where SSSS denotes four simply supported edges, SCSC denotes two simply supported opposite edges and two clamped opposite edges, CCCC denotes four clamped edges. Taking the laminate in Fig. $4 \mathrm{~b}$ as an example, the boundary condition can be written as

$v=w=0, \quad \sigma_{x}=0 \quad$ on $x=0, \quad x=A$

simply supported edges

$u=v=w=0 \quad$ on $y=0, \quad y=B \quad$ clamped edges

Laminates with a cyclic periodicity seldom occur in practice, but fortunately, in many cases of practical importance, an equivalent structure with such a characteristic can be introduced.

Take the laminate in Fig. $4 \mathrm{a}$ as an example. The laminate is divided into $m \times n$ uniform equivalent 4 -node elements (see Fig. 3 )

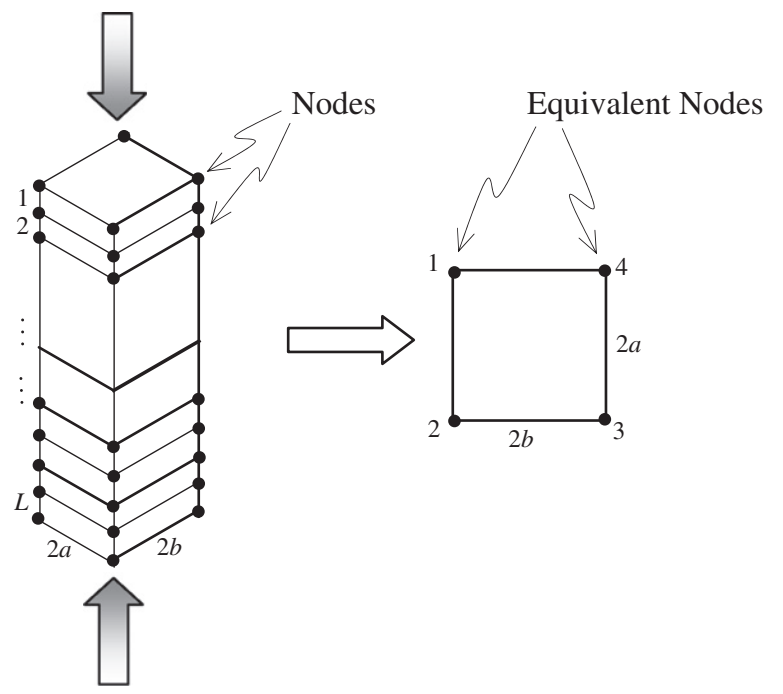

Fig. 3. An equivalent 2-D 4-node element.



(a)

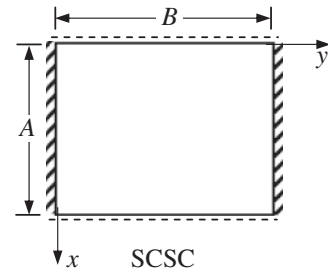

(b)

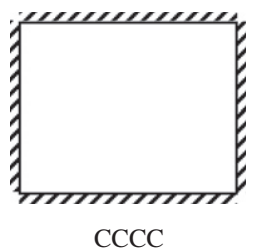

(c)
Fig. 4. Laminates with different boundary conditions: (a) four edges simply supported (SSSS), (b) two opposite edges simply supported and two opposite edges clamped (SCSC), (c) four edges clamped (CCCC).

as shown in Fig. 5a. The original laminate does not possess a cyclic periodicity. Extend the laminate in Fig. 5a twice by producing an anti-symmetrical image about the right edge and lower edge, respectively, as shown in Fig. 5c. The equivalent structure is a new rectangular laminate with four simply supported edges, whose displacements (deflections and rotations) on each set of opposite edges have equal values. Hence each set of opposite edges can be imaginarily joined. Correspondingly, the equivalent system consists of $2 m \times 2 n$ uniform equivalent 4 -node elements, whose each couple of equivalent nodes on the opposite edges can be imaginarily put together and treated as one node. In this sense, the equivalent system is without ends, and consists of $2 m \times 2 n$ repeating equivalent 4 -node elements and $2 m \times 2 n$ repeating equivalent nodes. According to [20], such an equivalent system can be regarded as a cyclic periodic structure in two directions, and can be decoupled in the unitary space. In the case of four edges simply supported, the boundary condition of the original laminate will be satisfied automatically in the equivalent system and supports may be removed.

Referring to Fig. 5b and c, the continuity across the nodes requires the following conditions to be satisfied:

$$
\begin{aligned}
& \{\delta\}_{2(j, k)}^{\mathrm{EN}}=\{\delta\}_{1(j+1, k)}^{\mathrm{EN}}, \quad\{\delta\}_{3(j, k)}^{\mathrm{EN}}=\{\delta\}_{1(j+1, k+1)}^{\mathrm{EN}}, \\
& \{\delta\}_{4(j, k)}^{\mathrm{EN}}=\{\delta\}_{1(j, k+1)}^{\mathrm{EN}} \quad(j=1,2, \ldots, 2 m ; k=1,2, \ldots, 2 n)
\end{aligned}
$$

The cyclic periodicity in two directions requires the following coupling conditions of the boundary nodes of the equivalent system in Fig. $5 c$ to be satisfied:

$$
\begin{aligned}
& \{\delta\}_{1(2 m+1, k)}^{\mathrm{EN}}=\{\delta\}_{1(1, k)}^{\mathrm{EN}}, \quad\{\delta\}_{1(2 m+1,2 n+1)}^{\mathrm{EN}}=\{\delta\}_{1(1,1)}^{\mathrm{EN}}, \\
& \{\delta\}_{1(j, 2 n+1)}^{\mathrm{EN}}=\{\delta\}_{1(j, 1)}^{\mathrm{EN}}
\end{aligned}
$$

Move on to the laminates in Fig. $4 \mathrm{~b}$ and c. Remove the constraints about the rotations and add corresponding distributed couples, the laminates in Fig. $4 \mathrm{~b}$ and $\mathrm{c}$ is changed into ones with four simply supported edges subjected to unknown distributed couples on the boundaries. In 3-D analysis the distributed couples consist of distributed forces on the boundary sections. Thus for the laminates in Fig. 4b and c, equivalent structures with a doubly cyclic periodicity can be introduced and they are shown in Fig. 6a and b, respectively.

The potential energy of the extended laminate shown in Fig. $5 \mathrm{c}$ may be expressed as

$\Pi=\sum_{j=1}^{2 m} \sum_{k=1}^{2 n} \pi_{j k}$

where $\pi_{j k}$ represents the potential energy of an equivalent 4-node element (EE), and $j, k$ are the element serial numbers in $x$ and $y$ directions, respectively. The potential energy for the element $(j, k)$ can be further expressed as 


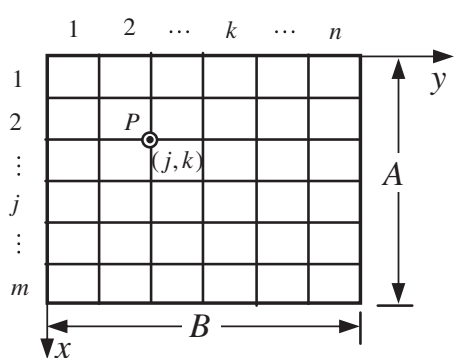

(a)

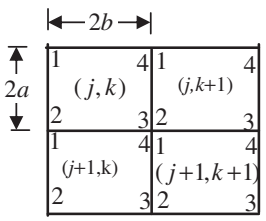

(b)

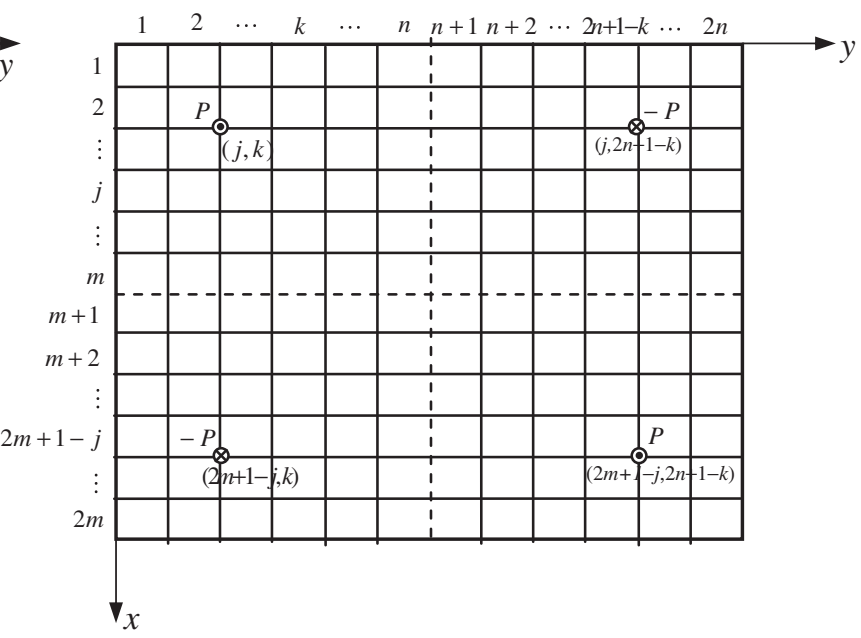

(c)

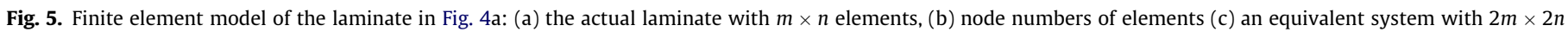
elements.

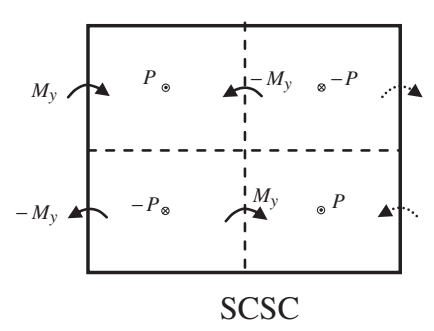

(a)

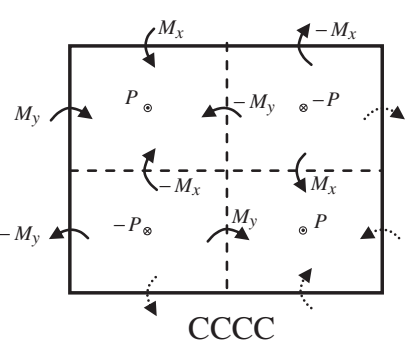

(b)
Fig. 6. The equivalent structures of the laminates in Fig. $4 \mathrm{~b}$ and c.

$$
\begin{aligned}
\pi_{j k} & =\frac{1}{2}\left(\{\delta\}_{(j, k)}^{\mathrm{EE}}\right)^{\mathrm{T}}[K]^{\mathrm{EE}}\{\delta\}_{(j, k)}^{\mathrm{EE}}-\left(\{F\}_{(j, k)}^{\mathrm{EE}}\right)^{\mathrm{T}}\{\delta\}_{(j, k)}^{\mathrm{EE}} \\
& =\frac{1}{2}\left(\{\bar{\delta}\}_{(j, k)}^{\mathrm{EE}}\right)^{\mathrm{T}}[K]^{\mathrm{EE}}\{\delta\}_{(j, k)}^{\mathrm{EE}}-\frac{1}{2}\left(\left(\{\bar{\delta}\}_{(j, k)}^{\mathrm{EE}}\right)^{\mathrm{T}}\{F\}_{(j, k)}^{\mathrm{EE}}+\left(\{\bar{F}\}_{(j, k)}^{\mathrm{EE}}\right)^{\mathrm{T}}\{\delta\}_{(j, k)}^{\mathrm{EE}}\right)
\end{aligned}
$$

where $[K]^{\mathrm{EE}},\{F\}_{(j, k)}^{\mathrm{EE}}$ and $\{\delta\}_{(j, k)}^{\mathrm{EE}}$ denote the stiffness matrix, loading and displacement vectors of the equivalent 4-node element numbered $(j, k)$, respectively. The superior bar denotes complex conjugation, which may be not necessary for real variables, but according to [20] such a complex conjugation expression is much convenient for deriving the variational equation with complex variables.

\section{Decoupling of finite element matrix equations by applying the U-transformation} as

The conventional finite element matrix equation can be written

$\mathbf{K} \boldsymbol{\delta}=\mathbf{F}$

where $\mathbf{K}, \boldsymbol{\delta}$ and $\mathbf{F}$ are the total stiffness matrix, node displacement vector and load vector, respectively. For 3-D analysis of a laminate, the total stiffness matrix $\mathbf{K}$ is very large. If elements possess a doubly cyclic periodicity, the total stiffness matrix can be a quasi-diagonal matrix, and the finite element matrix equation can be decoupled by applying the U-transformation (refer to Appendix). Noting Eq. (A.10) in Appendix, Eq. (8) premultiplied by $\overline{\mathbf{U}}_{r s}^{\mathrm{T}}$ becomes
$\overline{\mathbf{U}}_{r s}^{\mathrm{T}} \mathbf{K} \mathbf{U}_{r s} \overline{\mathbf{U}}_{r s}^{\mathrm{T}} \boldsymbol{\delta}=\overline{\mathbf{U}}_{r s}^{\mathrm{T}} \mathbf{F}$

For the problem in Fig. 5c, $\delta$ can be expressed as:

$\boldsymbol{\delta}=\left[\{\delta\}_{1(j, k)}^{\mathrm{EN}}\right] \quad(j=1,2, \ldots, 2 m ; k=1,2, \ldots, 2 n)$

Let

$\mathbf{q}_{r s}=\overline{\mathbf{U}_{r s}^{\mathrm{T}} \boldsymbol{\delta} ;} \quad \mathbf{f}_{r s}=\overline{\mathbf{U}}_{r s}^{\mathrm{T}} \mathbf{F}$

which can be expressed in component form

$\mathbf{q}_{r s}=\frac{1}{\sqrt{2 m} \sqrt{2 n}} \sum_{j=1}^{2 m} \sum_{k=1}^{2 n} \mathrm{e}^{-\mathrm{i}(j-1) r \psi_{1}} \mathrm{e}^{-\mathrm{i}(k-1) s \psi_{2}} \boldsymbol{\delta}_{j k}$
$\mathbf{f}_{r s}=\frac{1}{\sqrt{2 m} \sqrt{2 n}} \sum_{j=1}^{2 m} \sum_{k=1}^{2 n} \mathrm{e}^{-\mathrm{i}(j-1) r \psi_{1}} \mathrm{e}^{-\mathrm{i}(k-1) s \psi_{2}} \mathbf{F}_{j k}$

where $\psi_{1}=2 \pi / 2 m, \psi_{2}=2 \pi / 2 n$. Then the decoupled matrix equation in the unitary space is obtained

$\mathbf{k}_{r s} \mathbf{q}_{r s}=\mathbf{f}_{r s} \quad(r=1,2, \ldots, 2 m ; s=1,2, \ldots, 2 n)$

The matrix equation (Eq. (8)) of $2 m \times 2 n \times(L+1) \times 3$-order is decoupled into $2 m \times 2 n$ individual matrix equations of $(L+1) \times$ 3 -order. As an actual case, the original laminate in Fig. 5(a) is divided into $m \times n$ equivalent 4-node elements and the equivalent system in Fig. 5(c) contains $2 m \times 2 n$ equivalent 4-node elements. The total matrix equation is decoupled to $2 m \times 2 n$ individual matrix equations. Due to the anti-symmetry of the equivalent system, one needs to solve only $m \times n$ individual matrix equations of $(L+1) \times 3$-order in the unitary space.

In actual computation, it is not necessary to decouple the total stiffness matrix as above. Chan et al. [20] present a more direct method to decouple the governing equations, of course the principle is the same.

Applying the U-transformation to the displacement and load vectors, one obtains

$\{\delta\}_{(j, k)}^{\mathrm{EE}}=\frac{1}{\sqrt{2 m} \sqrt{2 n}} \sum_{r=1}^{2 m} \sum_{s=1}^{2 n} \mathrm{e}^{\mathrm{i}(j-1) r \psi_{1}} \mathrm{e}^{\mathrm{i}(k-1) s \psi_{2}}\{q\}_{(r, s)}^{\mathrm{EE}}$

$\{F\}_{(j, k)}^{\mathrm{EE}}=\frac{1}{\sqrt{2 m} \sqrt{2 n}} \sum_{r=1}^{2 m} \sum_{s=1}^{2 n} \mathrm{e}^{\mathrm{i}(j-1) r \psi_{1}} \mathrm{e}^{\mathrm{i}(k-1) s \psi_{2}}\{f\}_{(r, s)}^{\mathrm{EE}}$ yields

The substitution of Eqs. (15) and (16) into Eqs. (5a) and (5b) 
$\{q\}_{2(r, s)}^{\mathrm{EN}}=\mathrm{e}^{\mathrm{i} r \psi_{1}}\{q\}_{1(r, s)}^{\mathrm{EN}}, \quad\{q\}_{3(r, s)}^{\mathrm{EN}}=\mathrm{e}^{\mathrm{i}\left(r \psi_{1}+s \psi_{2}\right)}\{q\}_{1(r, s)}^{\mathrm{EN}}$,

$\{q\}_{4(r, s)}^{\mathrm{EN}}=\mathrm{e}^{\mathrm{i} s \psi_{2}}\{q\}_{1(r, s)}^{\mathrm{EN}}$

which can also be expressed as

$\{q\}_{(r, s)}^{\mathrm{EE}}=[T]_{(r, s)}\{q\}_{1(r, s)}^{\mathrm{EN}}, \quad[T]_{(r, s)}=\left[\begin{array}{llll}\mathbf{I} & e^{\mathrm{i} r \psi_{1} \mathbf{I}} & e^{\mathrm{i}\left(r \psi_{1}+s \psi_{2}\right)} \mathbf{I} & e^{\mathrm{i} s \psi_{2} \mathbf{I}}\end{array}\right]$

where I is a $3(L+1) \times 3(L+1)$ identity matrix. The substitution of Eq. (18) into Eqs. (15) and (16), and then into Eqs. (6) and (7) yields the potential energy in the unitary space, whose variation equation results in

$[k]_{1(r, s)}^{\mathrm{EN}}\{q\}_{1(r, s)}^{\mathrm{EN}}=\{f\}_{1(r, s)}^{\mathrm{EN}} \quad(r=1,2, \ldots, 2 m ; s=1,2, \ldots, 2 n)$

where

$[k]_{1(r, s)}^{\mathrm{EN}}=[\bar{T}]_{(r, s)}^{\mathrm{T}}[K]^{\mathrm{EE}}[T]_{(r, s)}, \quad\{f\}_{1(r, s)}^{\mathrm{EN}}=[\bar{T}]_{(r, s)}^{\mathrm{T}}\{f\}_{(r, s)}^{\mathrm{EE}}$

$[\bar{T}]_{(r, s)}^{\mathrm{T}}$ denotes the conjugate transposed matrix of $[T]_{(r, s)}$. Let

$[\rho]_{1(r, s)}^{\mathrm{EN}}=\left([k]_{1(r, s)}^{\mathrm{EN}}\right)^{-1}$

Then the displacement vector of the equivalent nodes in the unitary space can be expressed as

$\{q\}_{1(r, s)}^{\mathrm{EN}}=[\rho]_{1(r, s)}^{\mathrm{EN}}\{f\}_{1(r, s)}^{\mathrm{EN}} \quad(r=1,2, \ldots, 2 m ; s=1,2, \ldots, 2 n)$

Transform it into the actual nodal displacement vector

$\{\delta\}_{1(j, k)}^{\mathrm{EN}}=\frac{1}{\sqrt{2 m} \sqrt{2 n}} \sum_{r=1}^{2 m} \sum_{s=1}^{2 n} \mathrm{e}^{\mathrm{i}(j-1) r \psi_{1}} \mathrm{e}^{\mathrm{i}(k-1) s \psi_{2}}\{q\}_{1(r, s)}^{\mathrm{EN}}$

in terms of which the actual displacement, strain and stress fields of the laminate can be obtained.

\section{Load vectors in the unitary space for laminates with different boundary conditions}

The total loads on the equivalent system of a laminate contain equivalent external loads (the actual loads and their images) and constraint loads (the distributed constraint couples and their images) as shown in Fig. 5c and Fig. 6. So the total load vector in the unitary space, $\{f\}_{1(r, s)}^{\mathrm{EN}}$, consists of two parts

$\{f\}_{1(r, s)}^{\mathrm{EN}}=\left\{f^{\mathrm{EL}}\right\}_{1(r, s)}^{\mathrm{EN}}+\left\{f^{\mathrm{CL}}\right\}_{1(r, s)}^{\mathrm{EN}}$

where $\left\{f^{\mathrm{EL}}\right\}_{1(r, s)}^{\mathrm{EN}}$ and $\left\{f^{\mathrm{CL}}\right\}_{1(r s)}^{\mathrm{EN}}$ are corresponding to the equivalent external loads (EL) and constraint loads (CL), and they are dealt with in Sections 4.1 and 4.2, respectively.

\subsection{Equivalent external load vector in the unitary space}

As illustrative examples, consider two actual external loads.

(1) Uniform transverse external load $p_{0}$

The actual load and its images on the equivalent system can be expresses by the following function $p(x, y)$

$$
\begin{aligned}
& p(x, y) \\
& \quad= \begin{cases}p_{0} & 0 \leqslant x<A \text { and } 0 \leqslant y<B \text { or } A \leqslant x<2 A \text { and } B \leqslant y<2 B \\
-p_{0} & A \leqslant x<2 A \text { and } 0 \leqslant y<B \text { or } 0 \leqslant x<A \text { and } B \leqslant y<2 B\end{cases}
\end{aligned}
$$

The load in Eq. (25) is discretized to the finite element nodes, then by using the U-transformation (Eq. (13)), the load vector in the unitary space can be expressed as

$$
\begin{aligned}
& \left\{f^{\mathrm{EL}}\right\}_{1(r, s)}^{\mathrm{EN}}=\frac{8 p_{0} a b}{\sqrt{m n}}\left\{\left\{0,0,-\cot \frac{r \psi_{1}}{2} \cot \frac{S \psi_{2}}{2}\right\}^{\mathrm{T}},\right. \\
& \underbrace{\{0,0,0\}^{\mathrm{T}},\{0,0,0\}^{\mathrm{T}}, \cdots,\{0,0,0\}^{\mathrm{T}}}_{L}\} \\
& (r=1,3, \ldots, 2 m-1 \text { and } s=1,3, \ldots, 2 n-1) \\
& \left\{f^{\mathrm{EL}}\right\}_{1(r, s)}^{\mathrm{EN}}=\mathbf{0} \quad(r=2,4, \ldots, 2 m \text { or } s=2,4, \ldots, 2 n) \\
& \text { where } \psi_{1}=2 \pi / 2 m, \psi_{2}=2 \pi / 2 n \text {. }
\end{aligned}
$$

(2) Bi-sinusoidal transverse load

In this loading case, it is seen that the load functions for both the original laminate and the corresponding equivalent system are the same

$p(x, y)=p_{0} \sin \frac{\pi x}{A} \sin \frac{\pi y}{B}$

where $0 \leqslant x \leqslant A, 0 \leqslant y \leqslant B$ on the original laminate and $0 \leqslant x \leqslant 2 A, 0 \leqslant y \leqslant 2 B$ on the equivalent system. By using Eq. (13), the load vectors in the unitary space can be expressed as

$$
\begin{aligned}
& \left\{f^{\mathrm{EL}}\right\}_{1(r, s)}^{\mathrm{EN}}=\frac{-32 p_{0} a b m^{3} n^{3}}{\pi^{4} \sqrt{m n}}\left\{\left\{0,0,\left(\sin \frac{\psi_{1}}{2} \sin \frac{\psi_{2}}{2}\right)^{2}\right\}^{\mathrm{T}},\right. \\
& \underbrace{\{0,0,0\}^{\mathrm{T}},\{0,0,0\}^{\mathrm{T}}, \cdots,\{0,0,0\}^{\mathrm{T}}}_{L}\} \\
& (r=1 \text { and } s=1 \text {, or } r=2 m-1 \text { and } s=2 n-1) \\
& \left\{f^{\mathrm{EL}}\right\}_{1(r, s)}^{\mathrm{EN}}=\frac{32 p_{0} a b m^{3} n^{3}}{\pi^{4} \sqrt{m n}}\left\{\left\{0,0,\left(\sin \frac{\psi_{1}}{2} \sin \frac{\psi_{2}}{2}\right)^{2}\right\}^{\mathrm{T}},\right. \\
& \underbrace{\{0,0,0\}^{\mathrm{T}},\{0,0,0\}^{\mathrm{T}}, \cdots,\{0,0,0\}^{\mathrm{T}}}_{L}\} \\
& (r=1 \text { and } s=2 n-1 \text {, or } r=2 m-1 \text { and } s=1) \\
& \left\{f^{\mathrm{EL}}\right\}_{1(r, s)}^{\mathrm{EN}}=\mathbf{0} \text { (for the rest of } \mathrm{r}, \mathrm{s} \text { ) }
\end{aligned}
$$

It is noted that the load vector in the unitary space contains only four nonzero terms which is much simpler than the corresponding load vector of the conventional finite element equation.

\subsection{Equivalent constraint load vector in the unitary space}

In the case of four simply supported edges (SSSS), $\left\{f^{\mathrm{CL}}\right\}_{1(r, s)}^{\mathrm{EN}}$ in Eq. (24) is equal to zero. However, for laminates with clamped edges, the equivalent systems as shown in Fig. 6 are subjected to unknown constraint couples and $\left\{f^{\mathrm{CL}}\right\}_{1(r, s)}^{\mathrm{EN}}$ requires determining.

Take the laminate with two simply supported opposite edges and two clamped opposite edges (SCSC) in Fig. 4b as an example, the displacement constraint conditions on the clamped edges are

$\left\{\delta_{y}\right\}_{1(j, 1)}^{\mathrm{EN}}=\left\{\delta_{y}\right\}_{1(j, n+1)}^{\mathrm{EN}}=\mathbf{0} \quad(j=1,2, \ldots, 2 m)$ on $y=0, y=B$

where $\left\{\delta_{y}\right\}_{1(j, n+1)}^{\mathrm{EN}}$ denotes all the components in $y$ direction of the displacement vector $\{\delta\}_{1(j, n+1)}^{\mathrm{EN}}$, i.e., the 2 th, 5 th, $\ldots,(3(L+1)-1)$ th components of $\{\delta\}_{1(j, n+1)}^{\mathrm{EN}}$. Only the constraint loads in $y$ direction remain due to the anti-symmetry of the equivalent system. The substitution of Eqs. (22) and (23) into Eq. (29) yields 


$$
\begin{aligned}
& \frac{1}{\sqrt{2 m} \sqrt{2 n}} \sum_{r=1}^{2 m} \mathrm{e}^{\mathrm{i}(j-1) r \psi_{1}} \sum_{s=1}^{2 n} \mathrm{e}^{\mathrm{i} s \pi}\left[\rho_{y,}\right]_{1(r, s)}^{\mathrm{EN}}\{f\}_{1(r, s)}^{\mathrm{EN}}=0 \\
& (j=1,2, \ldots, 2 m) \\
& \frac{1}{\sqrt{2 m} \sqrt{2 n}} \sum_{r=1}^{2 m} \mathrm{e}^{\mathrm{i}(j-1) r \psi_{1}} \sum_{s=1}^{2 n}\left[\rho_{y,}\right]_{1(r, s)}^{\mathrm{EN}}\{f\}_{1(r, s)}^{\mathrm{EN}}=0 \\
& (j=1,2, \ldots, 2 m)
\end{aligned}
$$

where $\left[\rho_{y}\right]_{1(r, s)}^{\mathrm{EN}}$ denotes the matrix consist of the 2th, 5 th, $\ldots,(3(L+1)-1)$ th rows of $[\rho]_{1(r, s)}^{\mathrm{EN}}$. As the matrix $\left[\mathrm{e}^{\mathrm{i}(j-1) r \psi_{1}}\right]$ $(r, j=1,2, \ldots, 2 m)$ is a full-rank matrix, the homogeneous system Eqs. (30a) and (30b) has only the trivial solution

$$
\begin{aligned}
& \sum_{s=1}^{2 n} \mathrm{e}^{\mathrm{i} s \pi}\left[\rho_{y},\right]_{1(r, s)}^{\mathrm{EN}}\left(\left\{f^{\mathrm{EL}}\right\}_{1(r, s)}^{\mathrm{EN}}+\left\{f^{\mathrm{CL}}\right\}_{1(r, s)}^{\mathrm{EN}}\right)=0 \\
& \sum_{s=1}^{2 n}\left[\rho_{y}\right]_{1(r, s)}^{\mathrm{EN}}\left(\left\{f^{\mathrm{EL}}\right\}_{1(r, s)}^{\mathrm{EN}}+\left\{f^{\mathrm{CL}}\right\}_{1(r, s)}^{\mathrm{EN}}\right)=0
\end{aligned}
$$

Explicit expressions of the constraint loads in the unitary space can be obtained as following:

(1) Uniform transverse external load

The substitution of Eq. (26) into Eqs. (31a) and (31b) yields

$$
\left\{f^{\mathrm{CL}}\right\}_{1(r, s)}^{\mathrm{EN}}= \begin{cases}-\left\{f^{\mathrm{CL}}\right\}_{1(r)}^{\mathrm{EN}} & r=1,3, \ldots, 2 m-1 \text { and } \\ & s=1,3, \ldots, 2 n-1 \\ \mathbf{0} & r=2,4, \ldots, 2 m\end{cases}
$$

where

$$
\begin{aligned}
\left\{f_{y}^{\mathrm{CL}}\right\}_{1(r)}^{\mathrm{EN}}= & \left(\sum_{s=1,3}^{2 n-1}\left[\rho_{y, y}\right]_{1(r, s)}^{\mathrm{EN}}\right)^{-1} \sum_{s=1,3}^{2 n-1}\left[\rho_{y,}\right]_{1(r, s)}^{\mathrm{EN}}\left\{f^{\mathrm{EL}}\right\}_{1(r, s)}^{\mathrm{EN}} \\
& (r=1,3, \ldots, 2 m-1)
\end{aligned}
$$

where $\left[\rho_{y, y}\right]_{1(r, s)}^{\mathrm{EN}}$ denotes the matrix consisting of the 2th, 5 th, $\ldots,(3(L+1)-1)$ th rows, the 2 th, 5 th, .., $(3(L+1)-1)$ th columns of $[\rho]_{1(r, s)}^{\mathrm{EN}},\left\{f_{y}^{\mathrm{CL}}\right\}_{1(r)}^{\mathrm{EN}}$ denotes the vector consisting of the 2 th, 5 th, $\ldots,(3(L+1)-1)$ th components of $\left\{f^{\mathrm{CL}}\right\}_{1(r)}^{\mathrm{EN}}$.

(2) Bi-sinusoidal transverse load

The Substitution of Eq. (28) into Eqs. (31a) and (31b) yields

$$
\left\{f^{\mathrm{CL}}\right\}_{1(r, s)}^{\mathrm{EN}}= \begin{cases}-\left\{f^{\mathrm{CL}}\right\}_{1(r)}^{\mathrm{EN}} & r=1,2 m-1 \text { and } s=1,2 n-1 \\ \mathbf{0} & \text { for the rest of } r \text { and } s\end{cases}
$$

where

$$
\begin{aligned}
\left\{f_{y}^{\mathrm{CL}}\right\}_{1(r)}^{\mathrm{EN}}= & \left(\sum_{s=1,3}^{2 n-1}\left[\rho_{y, y}\right]_{1(r, s)}^{\mathrm{EN}}\right)^{-1} \sum_{s=1,2 n-1}\left[\rho_{y,}\right]_{1(r, s)}^{\mathrm{EN}}\left\{f^{\mathrm{EL}}\right\}_{1(r, s)}^{\mathrm{EN}} \\
& (r=1,2 m-1)
\end{aligned}
$$

Similarly, the load vector in the unitary space for a laminate with four clamped edges (CCCC) can be obtained.

\section{Numerical results and comparison}

\begin{tabular}{|c|c|c|c|c|c|c|}
\hline \multirow[t]{2}{*}{$m^{\mathrm{a}}$} & \multirow[t]{2}{*}{$n^{\mathrm{a}}$} & \multicolumn{5}{|c|}{$\bar{w}(A / 2, B / 2,0)$} \\
\hline & & $l=2$ & $l=4$ & $l=8$ & $l=16$ & $l=32$ \\
\hline 10 & 10 & 1.97864 & 2.00211 & 2.00895 & 2.01072 & 2.01116 \\
\hline 20 & 20 & 1.97496 & 1.99819 & 2.00495 & 2.00670 & 2.00715 \\
\hline 40 & 40 & 1.97401 & 1.99719 & 2.00392 & 2.00567 & 2.00611 \\
\hline 80 & 80 & 1.97377 & 1.99693 & 2.00367 & 2.00541 & 2.00585 \\
\hline 160 & 160 & 1.97371 & 1.99687 & 2.00360 & 2.00535 & 2.00579 \\
\hline 320 & 320 & 1.97370 & 1.99685 & 2.00358 & 2.00533 & 2.00577 \\
\hline 640 & 640 & 1.97370 & 1.99685 & 2.00358 & 2.00532 & 2.00576 \\
\hline Exact solution [14] & & & & & & 2.00591 \\
\hline
\end{tabular}

The matrix equation of the U-transformation-finite element method is in a complex form, which can be solved by commercial software such as MATHEMATICA. In this section, several numerical examples and a comparison with available analytical and numeri-

\begin{tabular}{|c|c|c|c|c|c|c|}
\hline \multirow[t]{2}{*}{$m^{\mathrm{a}}$} & \multirow[t]{2}{*}{$n^{\mathrm{a}}$} & \multicolumn{5}{|c|}{$\bar{\tau}_{x z}(0, B / 2,0)$} \\
\hline & & $l=2$ & $l=4$ & $l=8$ & $l=16$ & $l=32$ \\
\hline 10 & 10 & 0.25008 & 0.25191 & 0.25251 & 0.25266 & 0.25270 \\
\hline 20 & 20 & 0.25248 & 0.25430 & 0.25490 & 0.25505 & 0.25509 \\
\hline 40 & 40 & 0.25308 & 0.25490 & 0.25549 & 0.25565 & 0.25569 \\
\hline 80 & 80 & 0.25323 & 0.25505 & 0.25564 & 0.25580 & 0.25584 \\
\hline 160 & 160 & 0.25327 & 0.25509 & 0.25568 & 0.25584 & 0.25588 \\
\hline 320 & 320 & 0.25328 & 0.25510 & 0.25569 & 0.25585 & 0.25589 \\
\hline 640 & 640 & 0.25328 & 0.25510 & 0.25569 & 0.25585 & 0.25589 \\
\hline Exact solution [14] & & & & & & 0.25590 \\
\hline
\end{tabular}
cal results are presented to demonstrate the accuracy and efficiency of the present method. In the following examples, the properties of the lamina are assumed to be

$$
\begin{aligned}
& E_{1}=172.4 \mathrm{GPa}\left(25 \times 10^{6} \mathrm{Psi}\right), \quad E_{2}=E_{3}=6.89 \mathrm{GPa}\left(10^{6} \mathrm{Psi}\right), \\
& G_{12}=G_{13}=3.45 \mathrm{GPa}\left(0.5 \times 10^{6} \mathrm{Psi}\right), \\
& G_{23}=1.378 \mathrm{GPa}\left(0.2 \times 10^{6} \mathrm{Psi}\right), \quad v_{12}=v_{13}=v_{23}=0.25
\end{aligned}
$$

Table 1

Convergence of nondimensional deflection $\bar{w}(A / 2, B / 2,0)$ of a square cross-ply laminate $\left(0^{\circ} / 90^{\circ} / 0^{\circ}\right)$ with four simply supported edges for the length-to-thickness ratio $s=4$.

a $m, n$ are the inplane mesh numbers, the nondimensional deflection refers to Eq. (35).

Table 2

Convergence of nondimensional stress $\bar{\tau}_{x z}(0, B / 2,0)$ of a square cross-ply laminate $\left(0^{\circ} / 90^{\circ} / 0^{\circ}\right)$ with four simply supported edges for the length-to-thickness ratio $s=4$.

${ }^{a} m, n$ are the inplane mesh numbers, the nondimensional stress refers to Eq. (35). 
where $E_{1}, E_{2}, E_{3}$ are the Young's moduli in three principal material directions, $G_{12}, G_{13}, G_{23}$ are the shear moduli, $v_{12}, v_{13}, v_{23}$ are the Poisson's ratios, respectively. For the convenience of comparison, the deflection $w$ and the shear stress $\tau_{x z}$ are nondimensionalized by

$\bar{w}=\frac{100 E_{2} w}{p_{0} H s^{4}}, \quad \bar{\tau}_{x z}=\frac{1}{p_{0} s} \tau_{x z}, \quad s=\frac{A}{H}$

where $p_{0}$ is a load parameter, $A$ is the edge length of a square laminate, $H$ is the thickness.

Example 1. Analyze a three-layer equi-thickness cross-ply $\left(0^{\circ} / 90^{\circ} /\right.$ $\left.0^{\circ}\right)$ square laminate $(A=B)$ with four simply supported edges (SSSS) under a bi-sinusoidal transverse load on the top surface (see Eq. (27)).

This is a benchmark problem and the exact elasticity solution $[14]$ is available. The variations of the nondimensional deflection $\bar{w}(A / 2, B / 2,0)$ and the nondimensional transverse shear stress

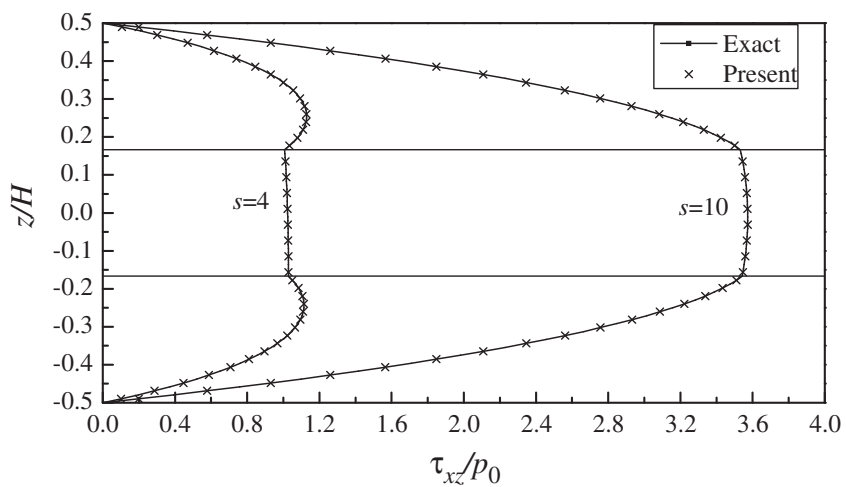

Fig. 7. Distributions of the nondimensional transverse shear stress $\tau_{x z} / p_{0}$ through the laminate thickness at the side midline for the length-to-thickness ratios $s=4$ and $s=10$ and a comparison with the exact elasticity solution [14]. $\bar{\tau}_{x z}(0, B / 2,0)$ with the element mesh numbers (inplane mesh $m \times n$, thickness mesh $l$ ) are listed in Tables 1 and 2 . It is seen that the present results are in almost exact agreement with the exact elasticity solution [14] when the number of the elements increases to $m \times n \times 3 \times l=80 \times 80 \times 3 \times 16$. For a thick laminate, the interlaminar transverse shear stress is a major concern since it may induce delamination failure. The distribution of the nondimensional transverse shear stress $\bar{\tau}_{x z}$ through the laminate thickness at the side midline for the length-to-thickness ratios $s=4$ and $s=10$ is shown in Fig. 7. It is observed that the present results and the exact solution cannot be distinguished in the figure. For a small lengthto-thickness, $s=4$, the maximum transverse shear stress appears in the facing layer and not in the core layer.

Example 2. Analyze a four-layer equi-thickness cross-ply $\left(0^{\circ} / 90^{\circ}\right.$ $90^{\circ} / 0^{\circ}$ and $0^{\circ} / 90^{\circ} / 0^{\circ} / 90^{\circ}$ )square laminate under a uniform load on the top surface and consider three boundary conditions: four simply supported edges (SSSS), two simply supported opposite edges and two clamped opposite edges (SCSC) and four clamped edges (CCCC).

Take a finite element mesh of $m \times n \times 4 \times l=80 \times 80 \times 4 \times 12$. The nondimensional deflections at the center on the top surface of laminates are listed in Table 3 for various length-to-thickness ratios. For a comparison, the exact elasticity solutions given by Pagano [14], as well as the numerical solutions by the 3-D FLM (3-D finite layer method) [15], the zigzag SFSM (Spline finite strip method) [8] and Reddy FSM (Finite strip method) [22] and Mindlin FSM [23] are also listed in Table 3. To our best knowledge, for laminates with non-simply supported edges, no exact 3-D elasticity solution was reported, and the present paper provides a full 3-D finite element solution. It should be noted that for the mesh of $m \times n \times 4 \times l=80 \times 80 \times 4 \times 12$, the number of degrees of freedom of the conventional finite element computations is $81 \times 81 \times(4 \times 12+1) \times 3=9.64 \times 10^{5}$, which is very large. After decoupling by the U-transformation method, the number of de-

Table 3

Nondimensional center deflection $\bar{w}(A / 2, B / 2,0)$ of a square cross-ply laminate with different boundary conditions under a uniform load by various methods.

\begin{tabular}{|c|c|c|c|c|c|c|c|}
\hline \multirow[t]{3}{*}{$s^{\mathrm{a}}$} & \multirow[t]{3}{*}{ Source } & \multicolumn{6}{|l|}{$\bar{w}(A / 2, B / 2,0)$} \\
\hline & & \multicolumn{2}{|l|}{$\mathrm{SSSS}^{\mathrm{b}}$} & \multicolumn{2}{|l|}{ SCSC $^{b}$} & \multicolumn{2}{|l|}{$\mathrm{CCCC}^{\mathrm{b}}$} \\
\hline & & $\left(0^{\circ} / 90^{\circ} / 90 / 0^{\circ}\right)$ & $\left(0^{\circ} / 90^{\circ} / 0^{\circ} / 90^{\circ}\right)$ & $\left(0^{\circ} / 90^{\circ} / 90^{\circ} / 0^{\circ}\right)$ & $\left(0^{\circ} / 90^{\circ} / 0^{\circ} / 90^{\circ}\right)$ & $\left(0^{\circ} / 90^{\circ} / 90^{\circ} / 0^{\circ}\right)$ & $\left(0^{\circ} / 90^{\circ} / 0^{\circ} / 90^{\circ}\right)$ \\
\hline \multirow[t]{6}{*}{4} & Elasticity [14] & 2.94005 & 2.96792 & - & - & - & - \\
\hline & Present & 2.9395 & 2.9674 & 2.2394 & 2.3817 & 1.9294 & 1.9930 \\
\hline & 3-DFLM[15] & 2.9688 & 3.0065 & - & - & - & - \\
\hline & Zigzag SFSM [8] & 2.9109 & 2.9788 & - & - & 1.5990 & 1.6733 \\
\hline & Reddy FSM [22] & 2.8676 & 2.4282 & - & - & 1.7387 & 1.5182 \\
\hline & Mindlin FSM [23] & 2.5674 & 2.3837 & - & - & 1.8418 & 1.7535 \\
\hline \multirow[t]{6}{*}{10} & Elasticity [14] & 1.14006 & 1.18167 & - & - & - & - \\
\hline & Present & 1.1400 & 1.1815 & 0.7505 & 0.7287 & 0.5300 & 0.5264 \\
\hline & 3-DFLM[15] & 1.1430 & 1.1852 & - & - & - & - \\
\hline & Zigzag SFSM [8] & 1.1405 & 1.1705 & - & - & 0.4937 & 0.4872 \\
\hline & Reddy FSM [22] & 1.1050 & 1.0693 & - & - & 0.4902 & 0.4145 \\
\hline & Mindlin FSM [23] & 1.0250 & 1.0597 & - & - & 0.4651 & 0.4271 \\
\hline \multirow[t]{6}{*}{20} & Elasticity [14] & 0.80289 & 0.90240 & - & - & - & - \\
\hline & Present & 0.8028 & 0.9023 & 0.5178 & 0.4127 & 0.2608 & 0.2692 \\
\hline & 3-DFLM[15] & 0.8035 & 0.9032 & - & - & - & - \\
\hline & Zigzag SFSM [8] & 0.8034 & 0.8993 & - & - & 0.2541 & 0.2599 \\
\hline & Reddy FSM [22] & 0.7925 & 0.8737 & - & - & 0.2475 & 0.2371 \\
\hline & Mindlin FSM [23] & 0.7694 & 0.8713 & - & - & 0.2341 & 0.2371 \\
\hline \multirow[t]{6}{*}{100} & Elasticity [14] & 0.68473 & 0.81231 & - & - & - & - \\
\hline & Present & 0.6847 & 0.8122 & 0.4408 & 0.2899 & 0.1490 & 0.1800 \\
\hline & 3-DFLM[15] & 0.6848 & 0.8123 & - & - & - & - \\
\hline & Zigzag SFSM [8] & 0.6848 & 0.8122 & - & - & 0.1487 & 0.1797 \\
\hline & Reddy FSM [22] & 0.6843 & 0.8112 & - & - & 0.1482 & 0.1786 \\
\hline & Mindlin FSM [23] & 0.6833 & 0.8111 & - & - & 0.1475 & 0.1761 \\
\hline
\end{tabular}

a $s$ is the length-to-thickness ratio of a laminate.

b SSSS denotes four simply supported edges, SCSC two simply supported opposite edges and two clamped opposite edges, CCCC four clamped edges. 
grees of freedom of each individual matrix equation is reduced to $(4 \times 12+1) \times 3=147$. From Table 3 , it is seen that for the laminate with four simply supported edges, the results of various methods verify each other and the present solutions are in the most excellent agreement with the exact elasticity solutions. For the laminate with four clamped edges, when the length-to-thickness ratio $s>20$, the results of various methods also verify each other, however, when $s=4$, there is a great difference. In this example, the present results are the largest. The reason may be that the present method is a full 3-D finite element analysis and there is no any superfluous constraint on the boundaries except the boundary displacement constraints $u_{0}=0, v_{0}=0, w_{0}=0$. Laminates with nonsimply supported edges require still further numerical researches.

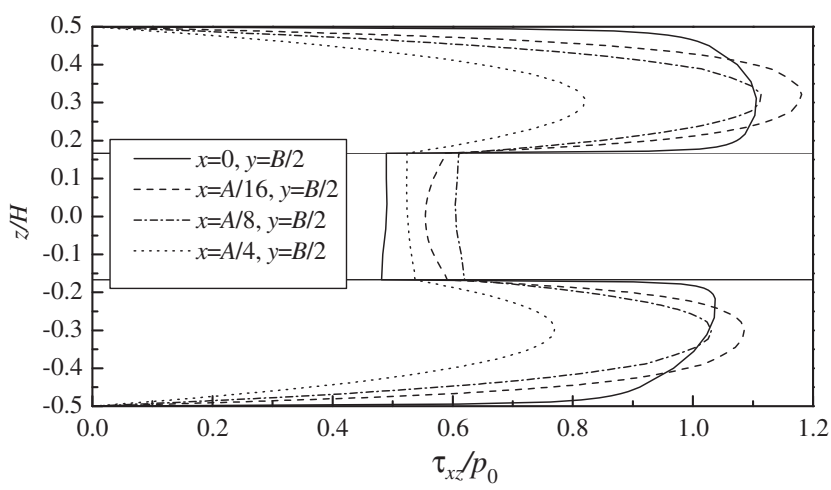

Fig. 8. Distributions of nondimensional transverse shear stress $\tau_{x z} / p_{0}$ through thickness at different locations in a three-layered laminate $\left(0^{\circ} / 90^{\circ} / 0^{\circ}\right)$ with all edges clamped (CCCC) for length-to-thickness $s=4$.

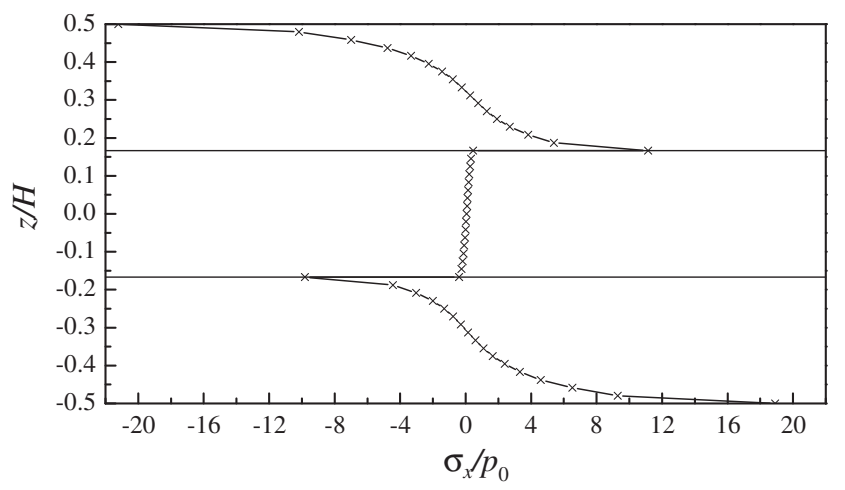

(a) $(x=0, y=B / 2)$

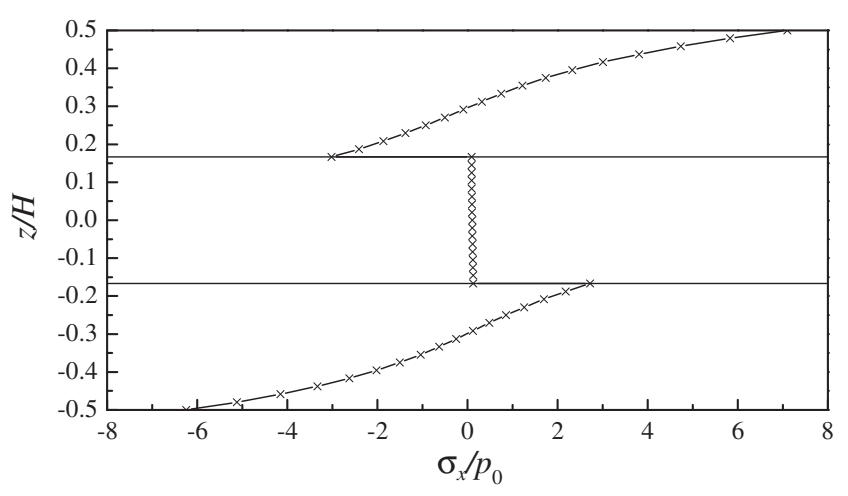

(b) $(x=A / 2, y=B / 2)$

Fig. 9. Variations of the nondimensional inplane normal stress $\sigma_{x} / p_{0}$ through the thickness of a clamped (CCCC) cross-ply $\left(0^{\circ} / 90^{\circ} / 0^{\circ}\right)$ square laminate with $s=4$ under a bi-sinusoidal transverse load: (a) $(x=0, y=B / 2)$; (b) $(x=A / 2, y=B / 2)$.
Example 3. Analyze a three-layer equi-thickness cross-ply $\left(0^{\circ} / 90^{\circ}\right.$ $0^{\circ}$ ) square laminate with four clamped edges (CCCC) and the length-to-thickness ratio, $s=4$, under a bi-sinusoidal transverse load (see Eq. (27)) on the top surface. Observe the distributions of the nondimensional transverse shear stress $\tau_{x z} / p_{0}$ and inplane normal stress $\sigma_{x} / p_{0}$ through the laminate thickness

Take a finite element mesh of $m \times n \times 3 \times l=80 \times 80 \times 3 \times 16$. The distributions of the nondimensional transverse shear stress $\tau_{x z} / p_{0}$ through the thickness $(x=0, A / 16, A / 8, A / 4 ; y=B / 2$. See Fig. 1) are depicted in Fig. 8. It is observed that the maximum value appears in the $0^{\circ}$ facing layer, which is similar to the result for the laminate with four simply supported edges. The transverse shear stress in the facing layer is much larger than that in the $90^{\circ}$ core layer and a sudden change occurs across the interface, which is different from the case of four simply supported edges. The distributions of the nondimensional inplane normal stress $\sigma_{x} / p_{0}$ through the thickness along the midline of the side section $(x=0, y=B / 2)$ and the center line of the laminate $(x=A / 4, y=B / 2)$ are shown in Fig. 9a and b, respectively. It is observed that the normal stresses making up the bending moment are borne by the facing layers almost completely. In each facing layer, the normal stress changes its sign. The maximum value of the stress on the side section is much larger than one along the center line of the laminate.

\section{Conclusions}

The U-transformation-finite element method is developed in 3dimensional analysis of composite laminates. A rectangular laminate with $m \times n \times L$ elements and $(m+1) \times(n+1) \times(L+1) \times 3$ degrees of freedom is extended to a cyclic periodic equivalent system with $2 m \times 2 n \times L$ elements. The total matrix equation is decoupled to $2 m \times 2 n$ individual matrix equations with $(L+1) \times 3$ degrees of freedom. Due to the anti-symmetry of the equivalent system, only $m \times n$ individual matrix equations require solving. The computational effort is effectively reduced.

For the benchmark problem, i.e. a three-layer equi-thickness cross-ply $\left(0^{\circ} / 90^{\circ} / 0^{\circ}\right)$ square laminate with four simply supported edges under a bi-sinusoidal transverse load on the top surface, the results of various available methods verify each other and the present solutions are in the most excellent agreement with the exact elasticity solutions. For the four-layer equi-thickness cross-ply $\left(0^{\circ} / 90^{\circ} / 90^{\circ} / 0^{\circ}\right.$ and $\left.0^{\circ} / 90^{\circ} / 0^{\circ} / 90^{\circ}\right)$ square laminates with four clamped edges and for the length-to-thickness ratio $s=4$ under a uniform load on the top surface, different methods (no exact elasticity solution) provide very different results. The present results are the largest, and the reason may be that the present method is a full 3-D finite element analysis and there is no any superfluous constraint on the boundaries. Laminates with nonsimply supported edges require still further numerical researches.

Illustrative numerical examples are provided to observe the distributions of stresses in the laminates.

\section{Acknowledgements}

The work is supported by the National Natural Science Foundation of China under Grant NNSFC 10972020, and The Open Fund of LNM.

\section{Appendix A}

The basic principle of the U-transformation method is briefly introduced in the following. More detailed introductions are available in Chan's work [20]. 
The U-transformation is a complex linear transformation. The transformation matrix may be a complex square matrix called $U$ matrix, which satisfies

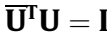

where I denotes the identity matrix, $\overline{\mathbf{U}}^{\mathrm{T}}$ denotes the conjugate transposed matrix of $\mathbf{U}$.

The $U$ matrix can be constructed as

$\mathbf{U} \equiv\left[\mathbf{U}_{1}, \mathbf{U}_{2}, \ldots, \mathbf{U}_{M}\right]$

$\mathbf{U}_{r}=\frac{1}{\sqrt{M}}\left[1, \mathrm{e}^{\mathrm{i} r \psi}, \mathrm{e}^{\mathrm{i} 2 r \psi}, \ldots, \mathrm{e}^{\mathrm{i}(M-1) r \psi}\right]^{\mathrm{T}} \quad(r=1,2, \ldots, M)$

with $\psi=2 \pi / M, \mathrm{i}=\sqrt{-1}$. It is easy to prove that $\mathbf{U}_{r}(r=1,2, \ldots, M)$ is an orthonormal basis.

Generally a Mdimensional square matrix $\mathbf{K}$ can be written as

$\mathbf{K}=\left[\begin{array}{cccc}k_{11} & k_{12} & \cdots & k_{1 M} \\ k_{21} & k_{22} & \cdots & k_{2 M} \\ \vdots & \vdots & \ddots & \vdots \\ k_{M 1} & k_{M 2} & \cdots & k_{M M}\end{array}\right]$

If $\mathbf{K}$ is a cyclic matrix, its components satisfy

$k_{11}=k_{22}=\cdots=k_{M M}$

$k_{1 j}=k_{2, j+1}=\cdots=k_{M-j+1, M}=k_{M-j+2,1}=\cdots=k_{M, j-1} \quad(j=2,3, \ldots, M)$

With the U matrix defined by Eq. (A.2), the cyclic matrix mentioned above can be transformed into the following diagonal matrix:

$\overline{\mathbf{U}}^{\mathbf{T}} \mathbf{K U}=\left[\begin{array}{llll}k_{1} & & & 0 \\ & k_{2} & & \\ & & \ddots & \\ 0 & & & k_{M}\end{array}\right]$

where

$k_{r}=\sum_{j=1}^{M} k_{1 j} \mathrm{e}^{\mathrm{i}(j-1) r \psi} \quad(r=1,2, \ldots, M)$

If the components $\mathbf{K}_{1 j}$ of the cyclic matrix $\mathbf{K}$ are also cyclic matrixes, generally expressed as

$\mathbf{K}_{1 j}=\left[\begin{array}{cccccc}\mathbf{K}_{1 j}^{(1,1)} & \mathbf{K}_{1 j}^{(1,2)} & \mathbf{K}_{1 j}^{(1,3)} & \cdots & \mathbf{K}_{1 j}^{(1, N-1)} & \mathbf{K}_{1 j}^{(1, N)} \\ \mathbf{K}_{1 j}^{(1, N)} & \mathbf{K}_{1 j}^{(1,1)} & \mathbf{K}_{1 j}^{(1,2)} & \ldots & \mathbf{K}_{1 j}^{(1, N-2)} & \mathbf{K}_{1 j}^{(1, N-1)} \\ \mathbf{K}_{1 j}^{(1, N-1)} & \mathbf{K}_{1 j}^{(1, N)} & \mathbf{K}_{1 j}^{(1,1)} & \ldots & \mathbf{K}_{1 j}^{(1, N-3)} & \mathbf{K}_{1 j}^{(1, N-2)} \\ \vdots & \vdots & \vdots & \ddots & \vdots & \vdots \\ \mathbf{K}_{1 j}^{(1,3)} & \mathbf{K}_{1 j}^{(1,4)} & \mathbf{K}_{1 j}^{(1,5)} & \ldots & \mathbf{K}_{1 j}^{(1,1)} & \mathbf{K}_{1 j}^{(1,2)} \\ \mathbf{K}_{1 j}^{(1,2)} & \mathbf{K}_{1 j}^{(1,3)} & \mathbf{K}_{1 j}^{(1,4)} & \ldots & \mathbf{K}_{1 j}^{(1, N)} & \mathbf{K}_{1 j}^{(1,1)}\end{array}\right]$

$(j=1,2, \ldots, M)$

where $\mathbf{K}_{1 j}^{(1, k)}$ can be a $J$ dimensional square matrix, the cyclic matrix $\mathbf{K}$ is a bi-cyclic matrix. It is necessary to construct two $U$ matrixes expressed as

$\mathbf{U}_{r}=\frac{1}{\sqrt{M}}\left[\mathbf{I}_{N \cdot J}, \mathbf{I}_{N \cdot J} \mathrm{e}^{\mathrm{i} r \psi_{1}}, \mathbf{I}_{N \cdot J} \mathrm{e}^{\mathrm{i} 2 r \psi_{1}}, \cdots, \mathbf{I}_{N \cdot J} \mathrm{e}^{\mathrm{i}(M-1) r \psi_{1}}\right]^{\mathrm{T}}$

$(r=1,2, \ldots, M)$

$\mathbf{U}_{s}=\frac{1}{\sqrt{N}}\left[\mathbf{I}_{J}, \mathbf{I}_{J} \mathrm{e}^{\mathrm{i} s \psi_{2}}, \mathbf{I}_{J} \mathrm{e}^{\mathrm{i} 2 s \psi_{2}}, \ldots, \mathbf{I}_{J} \mathrm{e}^{\mathrm{i}(N-1) s \psi_{2}}\right]^{\mathrm{T}} \quad(s=1,2, \ldots, N)$

with $\psi_{1}=2 \pi / M, \psi_{2}=2 \pi / N$, where $\mathbf{I}_{N \cdot J}$ denotes the identity matrix with $N \cdot J$ order, $\mathbf{I}_{J}$ denotes the identity matrix with $J$ order. With above two U matrixes, the bi-cyclic matrix can be transformed into a bi-diagonal matrix as follows

$\mathbf{k}_{r}=\overline{\mathbf{U}}_{r}^{\mathrm{T}} \mathbf{K} \mathbf{U}_{r}$

$\mathbf{k}_{r s}=\overline{\mathbf{U}}_{s}^{\mathrm{T}} \mathbf{K}_{r} \mathbf{U}_{s}=\overline{\mathbf{U}}_{s}^{\mathrm{T}} \overline{\mathbf{U}}_{r}^{\mathrm{T}} \mathbf{K} \mathbf{U}_{r} \mathbf{U}_{s}$

Let

$\mathbf{U}_{r s}=\mathbf{U}_{r} \mathbf{U}_{s}=\frac{1}{\sqrt{M}}\left[\mathbf{U}_{s}^{\mathrm{T}}, \mathbf{U}_{s}^{\mathrm{T}} \mathrm{e}^{\mathrm{i} r \psi_{1}}, \mathbf{U}_{s}^{\mathrm{T}} \mathrm{e}^{\mathrm{i} 2 r \psi_{1}}, \cdots, \mathbf{U}_{s}^{\mathrm{T}} \mathrm{e}^{\mathrm{i}(M-1) r \psi_{1}}\right]^{\mathrm{T}}$

It is easy to prove the following orthogonal properties

$\overline{\mathbf{U}}_{j k}^{\mathrm{T}} \mathbf{U}_{r s}=\delta_{j r} \delta_{k s} \mathbf{I}_{J} \quad j, r=1,2, \cdots, M ; \quad k, s=1,2, \cdots, N$

Then Eq. (A.8b) can be written as

$\mathbf{k}_{r s}=\overline{\mathbf{U}}_{r s}^{\mathrm{T}} \mathbf{K U}_{r s}$

or

$\mathbf{k}_{r s}=\sum_{j=1}^{M} \sum_{k=1}^{N} \mathbf{K}_{1 j}^{(1, k)} \mathrm{e}^{\mathrm{i}(j-1) r \psi_{1}} \mathrm{e}^{\mathrm{i}(k-1) s \psi_{2}}$

Above Eqs. (A.10) and (A.11) are the key of decoupling the finite element equations.

\section{References}

[1] Jones RM. Mechanics of composite materials. New York: McGraw-Hill; 1975.

[2] Medwadowski SJ. A refined theory of elastic orthotropic plates. ASME J App Mech 1958;25:437-43.

[3] Yang PC, Norris CH, Stavsky Y. Elastic wave propagation in heterogeneous plates. Int J Solids Struct 1966;2:665-84

[4] Reddy JN. A simple higher order theory for laminated composite plates. ASME J Appl Mech 1984;51:745-52.

[5] Phan ND, Reddy JN. Analysis of laminated composite plates using a higherorder shear deformation theory. Int J Numer Meth Eng 1985;21:2201-19.

[6] Reddy JN, Robbins DH. Theories and computational models for composite laminates. Appl Mech Rev 1994;47:147-69.

[7] Gay D, Hoa SV. Composite materials: design and applications. 2nd ed. New York: CRC Press; 2007

[8] Akhras G, Li W. Spline finite strip analysis of composite plates based on higherorder zigzag composite plate theory. Compos Struct 2007;78:112-8.

[9] Shi G. A new simple third-order shear deformation theory of plates. Int J Solids Struct 2007;44:4399-417.

[10] Swaminathan K, Patil SS. Higher order refined computational model with 12 degrees of freedom for the stress analysis of antisymmetric angle-ply plates analytical solutions. Compos Struct 2007;80:595-608.

[11] Robbins DH, Reddy JN. Modelling of thick composites using a layerwise laminate theory. Int J Numer Meth Eng 1993;36:655-77.

[12] Plagianakos TS, Saravanos DA. Higher-order layerwise laminate theory for the prediction of interlaminar shear stresses in thick composite and sandwich composite plates. Compos Struct 2009;87:23-35.

[13] Castro LMS, Ferreira AJM, Bertoluzza S, Batra RC, Reddy JN. A wavelet collocation method for the static analysis of sandwich plates using layerwise theory. Compos Struct 2010;92:1786-92.

[14] Pagano NJ. Exact solutions for rectangular bidirectional composites and sandwich plates. J Compos Mater 1970;4:20-34.

[15] Cheung YK, Jiang CP. Finite layer method in analyses of piezoelectric composite laminates. Comput Meth Appl Mech Eng 2001;191:879-901.

[16] Attallah KMZ, Ye JQ, Sheng HY. Three-dimensional finite strip analysis of laminated panels. Comput Struct 2007;85:1769-81.

[17] Bambole AN, Desai YM. Hybrid-interface element for thick laminated composite plates. Comput Struct 2007;85:1484-99.

[18] Kant T, Gupta AB, Pendhari SS, Desai YM. Elasticity solution for cross-ply composite and sandwich laminates. Compos Struct 2008;83:13-24.

[19] Cheung YK, Chan HC, Cai CW. Exact method for static analysis of periodic structures. ASCE J Eng Mech 1989;115:415-34.

[20] Chan HC, Cai CW, Cheung YK. Exact analysis of structures with periodicity using U-transformation. Singapore: World Scientific; 1998.

[21] Wilson EL, Taylor RL, Doherty WP, Ghaboussi J. Incompressible displacement modes. In: Fenves SJ, editor. Numerical and computer models in structura mechanics. New York: Academic Press; 1973.

[22] Akhras G, Cheung MS, Li W. Finite strip analysis of anisotropic laminated composite plates using higher-order shear deformation theory. Comput Struct $1994 ; 52: 471-7$

[23] Akhras G, Cheung MS, Li W. Static and vibration analysis of anisotropic composite laminates by finite strip method. Int J Solids Struct 1993;30:3129-37. 